\title{
Lenses of Extremely Wide Angle for Airplane Mapping
}

\section{By Irvine C. Gardner and Francis E. Washer}

\begin{abstract}
If the illumination of the image produced by a wide angle lens covering a field of 120 degrees follows the cosine-fourth-power law, the illumination at the edge of the field will be one-sixteenth that at the center in the absence of vignetting. By introducing negative distortion, the illumination may be expected to be more uniform from the center to the edge of the field. In particular, if there is no vignetting, and if the diaphragm prececes the lens, there will be uniform illumination, even for a field as great as 180 degrees, if the distortion is such that $r^{\prime}=f \sin \beta$, where $r^{\prime}$ is the distance from center of the field to a given image point, $\beta$ is the corresponding angular distance from the axis in the object space, and $f$ is the focal length corresponding to the part of the image in the neighborhood of the axis.

During the war Zeiss developed a mapping lens with a large amount of negative distortion and a second rectifying system by which undistorted copies could be obtained from the distorted negative made with the camera lens. A German mapping lens and a rectifying unit have been brought to this country, and detailed results of tests made at the National Bureau of Standards are given in this paper. The lens, termed the P'eon, covers a field of 130 degrees, and the distortion closely follows the law $r^{\prime}=f \beta$, the distortion being somewhat less than that of the preceding formula. Measurements of the resolving power, of the effective size of the entrance pupil for different angular distances from the axis, and of the net distortion of the two systems are reported. In a final print there is significant residual distortion resulting from the failure of the distortions of the two systems to exactly annul each other.
\end{abstract}

\section{Introduction}

In general, a distortion-free photographic lens forms an image on a plane surface over which the illumination conforms, at least approximately, to the cosine-fourth-power law. According to this law, the illumination, $E$, of the image at any point in the field when there is no vignetting, is given by the equation

$$
E=A_{0} \cos ^{4} \beta,
$$

where $A_{0}$ is the illumination at the axial point of the field and $\beta$ is the angle, measured in the object space, between the axis and a chief ray passing through the object point conjugate to the image point under consideration. This law seriously restricts the extent of field that can be usefully covered by a wide angle lens. To illustrate this, a 90 -degree field ( $\beta=45$-degrees) the ratio of the illumination at the edge of the field to that at the center is 0.25 in the absence of vignetting. Actually, with so large a field angle, vignetting is inevitable and the ratio is probably further reduced to the value 0.12 or possibly even less. If a useful field of 120-degrees were achieved, the ratio would be 0.06 before vignetting is considered. This decrease in illumination from the center outward is a serious disadvantage when using black-and-white emulsion and makes the use of color film, because of its more limited latitude, quite impossible. The illumination over the field can be made uniform by using a variable density filter a short distance in front of the lens and so designed that the illumination at the center of the field is reduced much more than that at the edge. Such a method is undesirable because it greatly reduces the effective speed of the lens and makes longer exposures necessary. Slusareff ${ }^{1}$ has called attention to the approximate character

\footnotetext{
1 G. Slusareff, J. Phys. USSR 4, 537 (1941); J. Opt. Soc. Am. 36, 707 (1946).
} 
of the cosine-four-power law and has cited a particular lens, designed by M. Russinoff, for which the illumination varies as the third power of the cosine. In this lens the image is free from distortion, and the desirable departure from the cosine-fourth-power law arises because the entrance pupil, which is conjugate to a diaphragm within the lens, is larger for an oblique entering beam than for an axial entering beam.

\section{Wide-Āngle Lenses With Negative Distortion}

It has been known for some time that a lens with a very large amount of negative distortion forms an image in which the illumination decreases much less rapidly from the center outward than is indicated by the cosine-fourth-power law. This is readily appreciated because, for the peripheral parts of the field, the luminous flux proceeding from a given small object area, for the lens with negative distortion, is concentrated on a region much smaller in area than for a distortionfree lens of the same equivalent focal length with consequent increase in the illumination. Gardner ${ }^{2}$ describes a photogrammetric method in which photographs are taken from an airplane with a wide angle lens by which large amounts of nega tive distortion are introduced and subsequently, by the use of a copying or projection lens, a final print is made that is free from distortion. Merte ${ }^{3}$ describes a wide-angle lens in which the first component is a negative meniscus. Distortion is not referred to in the patent, but computation shows that this lens does attain its wide coverage (180 degrees is claimed) by introducing large amounts of distortion. Richter, ${ }^{4}$ in the patent which evidently applies to the Pleon lens, describes two wide-angle systems, each of which consists of an achromatic negative meniscus system which introduces large amounts of distortion and a following positive system, relatively free from distortion, which forms a real image in the plane of the photographic emulsion. At present no information is available concerning United States or German patents assigned to the firm of Carl Zeiss and applying to the rectifying system used with the Pleon lens. In the patent literature that has just been discussed, there are

\footnotetext{
2 Irvine C. Gardner, U. S. Patent 2,037,017 (April 14, 1936).

3 W. Merte, U. S. Patent 2,126,126 (Aug. 9, 1938).

4 R. Richter, U. S. Patent 2,247,068 (June 24, 1941).
}

several statements concerning the more uniform illumination over the field of view resulting from the presence of large amounts of negative distortion. A quantitative statement of this effect has been given by Gardner ${ }^{5}$ and it is shown that if the diaphragm is in front of a photographic lens and if there is no vignetting, the field will be uniformly illuminated, even out to 180 degrees, provided that the distortion is such that

$$
r^{\prime}=f \sin \beta,
$$

where $r^{\prime}$ is the radial distance from the center of the field to a given object point, $f$ is the equivalent focal length corresponding to the scale of imagery in the neighborhood of the axis of the lens, and $\beta$ is as defined for 1. For a distortion-free lens

$$
r=f \tan \beta,
$$

and it is evident that the linear distortion corresponding to the angle $\beta$ in the true field is given by the equation

$$
\text { linear distortion }=f(\sin \beta-\tan \beta) .
$$

Applying eq 4 to a lens of $100-\mathrm{mm}$ focal length, the distortions corresponding to values of $\beta$ of 45 and 60 degrees are $29 \mathrm{~mm}$ (1.14 in.) and $87 \mathrm{~mm}$ (3.4 in.), respectively. These values illustrate the extremely large values of distortion that are encountered when uniformity of image illumination is secured by this means.

The lenses of extremely wide angle that have been referred to have the diaphragm within the lens system, and consequently eq 2 does not apply. However, the method by which eq 2 was derived can be applied to a lens system with an interna? diaphragm. For such a lens system the relation between distortion and uniformity of illumination must be determined by a computation applied to the design data of the lens under consideration, and no general statement can be made.

\section{Wide-Angle Photographs With Large Amounts of Distortion}

With the large amounts of distortion that have been considered it is evident that the scale of the photograph becomes rapidly smaller from the center outward, that straight lines not passing through the center become curved lines concave

${ }^{3}$ Irvine C. Gardner, J. Research NBS 39, 213 (1947) RP1824 
toward the center, and that the photographs cannot be assembled directly to form a mosaic. Such photographs may, however, be used in a variety of ways. In Germany the availability of photographs of this character led to the chance discovery that pilots of airplanes are not confused by such distortion if they are provided with photographs on which their location in flight is over the central portion of the picture. It was said that the outer, more distorted portion of the picture served as a "finder", and that the smaller scale, with accompanying loss of detail at the edge of the picture, suggests the loss of detail due to haze and increased distance when far off objects are viewed from a plane. The extremely wide angle lenses, however, were not developed to produce photographs for the use of aviators, and it is not known how extensively the photographs were used for this purpose.

The large amount of radial distortion does not present any obstacle to the use of the photographs, in their original form, for mapping by means of slotted templates or other methods of radial triangulation, although the large negative distortion and the smaller scale to which peripheral points are recorded lessen the precision with which the radial angles can be constructed or the slots cut. With the extremely large coverage of each photograph, it might be possible to orient a series of photographs with a minimum of ground control points, after which a sufficient number of additional points could be interpolated to serve as control for the use of narrower angle distortionfree photographs from which a final map can be constructed. For such a procedure to succeed it would probably be necessary to have a particularly favorable array of very conspicuous landmarks, easily identified from the air and ground, to serve as control points.

In general, however, when a lens of the type under consideration, with large field and large negative distortion, has been developed, it has been the intention to develop an accompanying copying or enlarging lens with a large amount of positive distortion so that the original distorted negative might be copied to produce an undistorted print. It has been mentioned that negative distortion tends to make the illumination more uniform from center to edge. By the same token, positive distortion acts to increase the nonuniformity of illumination beyond that pre- dicted by the cosine-fourth-power law, because the outer parts of the field, which in the natural course of events, would be more faintly illuminated than the center, have the illumination further decreased as a consequence of the greater magnification. For the copying lens, however, this unevenness of illumination is not so detrimental as in the case of the airplane camera lens, because copying is a laboratory process with the source of illumination entirely under control, and the uniformity of illumination in the image can be compensated by illuminating the negative in an appropriate manner.

One might envision the use of the copying lens of the preceding paragraph in a multiplex projector and the direct production of a contour map from the distorted photograph. The unevenness of illumination introduced by the projection lens, however, would introduce difficulties more serious for the multiplex operation than for the production of enlargements, and it appears that they might be insurmountable. On the other hand, the copying lens, instead of producing an enlargement, might be designed to produce a distortion-free diapositive of suitable scale to be used in a multiplex projector.

\section{Pleon Lens}

During World War II, Richter, of Zeiss, designed a camera lens and a rectifying lens of the type under consideration. They were designated, respectively, the Pleon lens and the Pleon rectifier. Samples of the lenses and drawings of the optical system were obtained by the intelligence groups working in Germany after the war. Figure 1 is a reproduction from a photograph of the Pleon lens tested. Figure 2 shows the optical system as specified (fig. 2 and table 2 ) in U. S. Patent $2,247,068$ (June 24, 1941). The first two elements form the member $A$, which is an achromatized lens of the type sometimes referred to as a "field compressor." The lens is designed to cover a full field of 130 degrees. The extreme chief rays entering the negative system and including this angle, after refraction, include an angle approximately 40.7 degrees. (These values are based on ray tracing applied to the design data given in the patent.) This smaller field is then imaged by the second member of the system at $B$, which is similar to the Metrogen lens in design. The diaphragm is at $C$, and $D$ represents a filter 


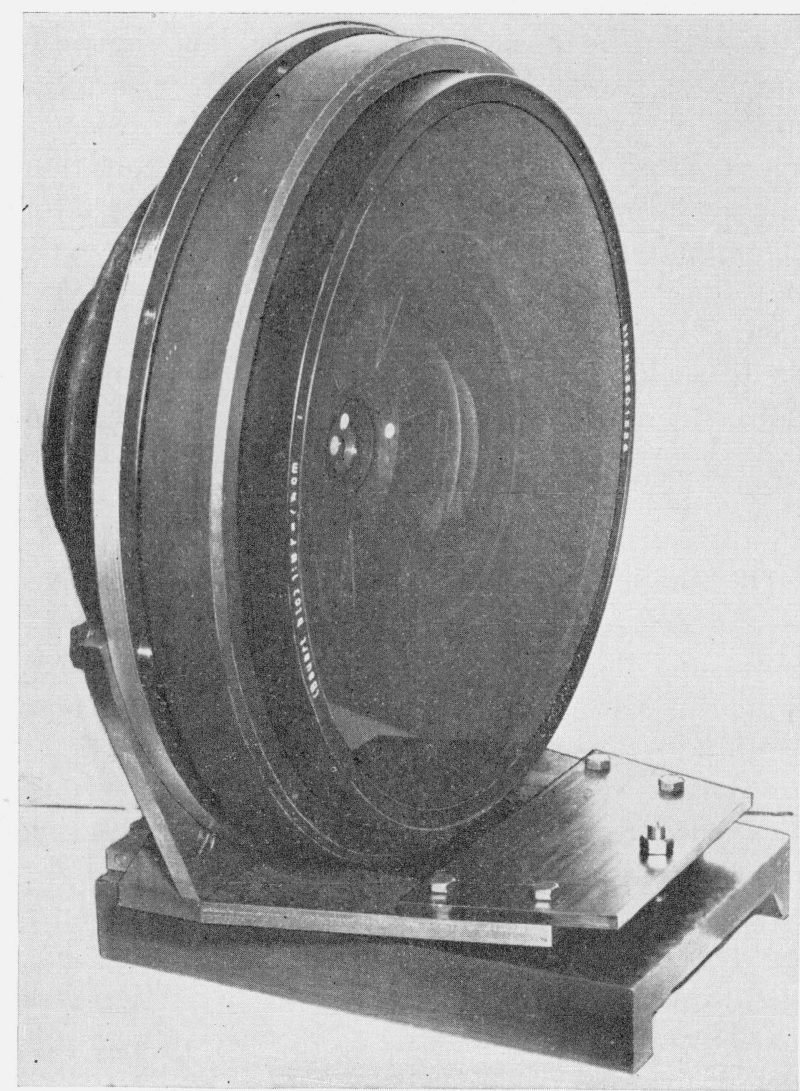

Figure 1. Pleon lens as mounted on a laboratory support for testing.

The surface shown is the large first surface of the field compressor and is turned toward the object. The diameter of this surface is approximately 1 foot.

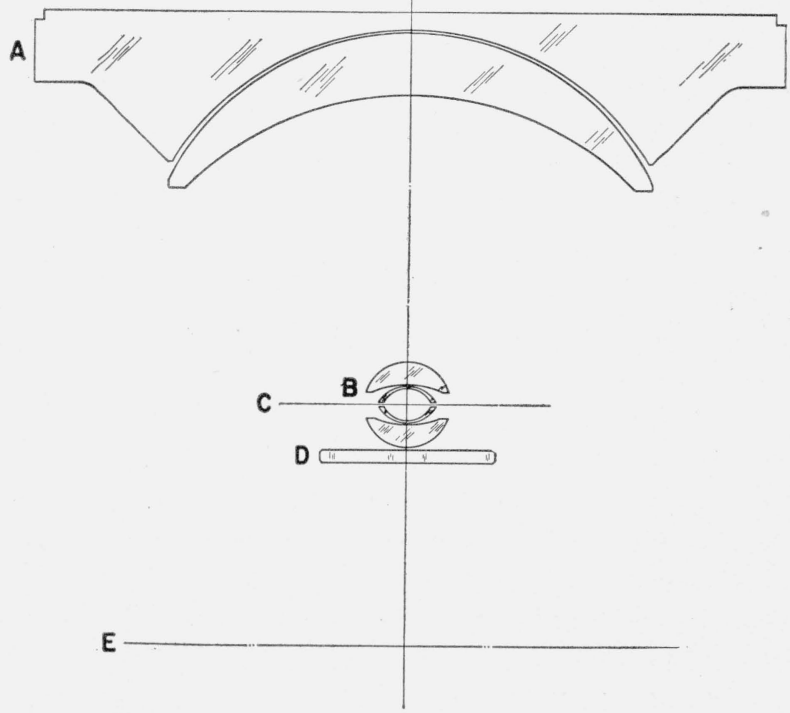

Figure 2. Optical system of the Pleon lens.

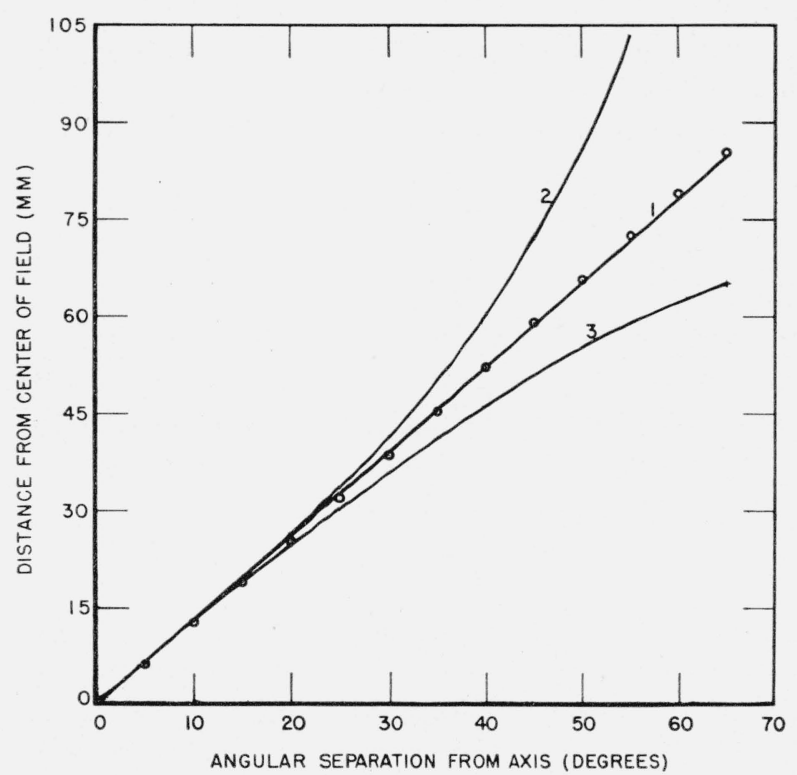

FIGURE 3. Relation between distance of image point from center of negative and angular displacement of corresponding object point from the axis for the Pleon lens.

Curve 1 shows results of measurements made on Pleon lens and corresponds respectively to equation $r=f \beta$. Curves 2 and 3 correspond respectively to equation $r^{\prime}=\tan \boldsymbol{\beta}$ applying to a distortion free lens, and to the equation $r^{\prime}=f \sin \beta$

which is an integral part of the lens. The image plane is at $E$. To give an idea of the size of this lens it may be noted that for a nominal focal length of $72.5 \mathrm{~mm}$, the over-all diameter of the first negative component is $300 \mathrm{~mm}$. and the distance from the plane surface of this component to the second surface of the filter $D$ is $180 \mathrm{~mm}$. In another method of considering this system. the negative member $A$ and a portion of the positive power of the second member may be assumed to constitute a Galilean telescopic system used backward, which produces an image at an infinite distance but much reduced in angular dimensions. The remaining power of the second member is utilized to form a real image on the photographic emulsion of this intermediate reduced image. The measured equivalent focal length of the camera lens, corresponding to the center of the field, is $72.1 \mathrm{~mm}$. The radius of the negative, corresponding to a half angle of approximately 65 degrees, is $85 \mathrm{~mm}$, and the linear distortion is $-68.8 \mathrm{~mm}$. In other words, in the absence of distortion, the radius of the negative corresponding to 65 degrees and the given focal length would be $154 \mathrm{~mm}$ from eq 3 . The distortion is such that 


$$
r^{\prime}=f \beta
$$

where the notation is the same as for eq 2 and 3 . This relationship is illustrated by the graph of figure 3. Curves corresponding to the relations, $r=f \tan \beta$ (see eq 3) and $r^{\prime}=f \sin \beta$ (see eq 2) are also shown in figure 3. The distortion, therefore, is not as great as that of er 2, but no direct deduction concerning the illumination can be made because the diaphragm is not in front of the lens system, as was specified in the example of section III. Experience shows that the illumination of the field is satisfactorily uniform. The resolving power is excellent, ranging from 82 lines per millimeter at the axis to 18 and 29 for tangential and radial lines, respectively, at 65 degrees.

\section{Pleon Rectifier}

Two views of the Pleon rectifier are shown in figures 4 and 5 . Figure 4 shows the instrument

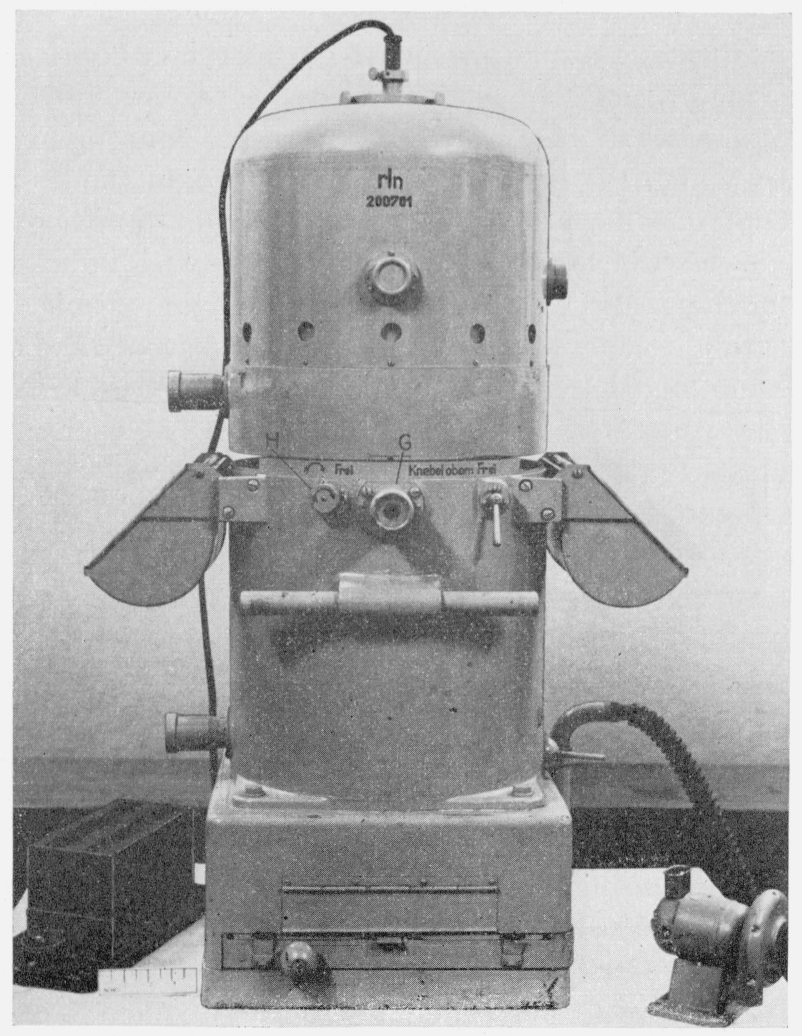

Figure 4. Pleon rectifier.

The film to be printed passes through the instrument near the center, the rolls of film resting in the metal pockets on either side. The paper is held flat near the bottom of the instrument by a vacuum back actuated by the small motor-driven exhaust pump at the right. The eyepiece, marked $G$, is part of an auxiliary optical system for centering the negative. The knob, $H$, swings four prisms into position for viewing the collimation marks on the negative in order to center it.

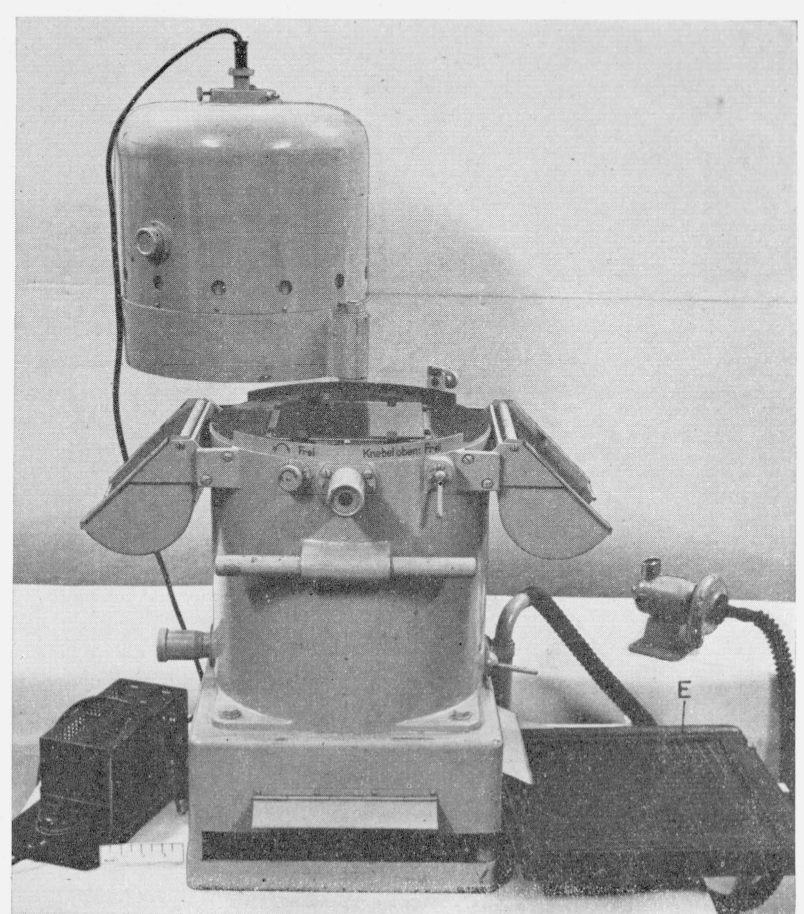

Figure 5. Pleon rectifier.

The top of the instrument is swung to the left to expose the glass pressure plate by which the negative film is held flat during the operation of printing. The sensitive paper is held flat by the vacuum plate swung out of the instrument in position for loaring. The two small doors near the bottom are for the insertion of paddles for dodging the print to compensate for differential illumination of the terrain at the time the exposure was made.

closed in position for making the rectified print. The film to be printed passes through the instrument near the center, the rolls of film resting in the metal pockets on either side. The paper is held flat near the bottom of the instrument by a vacuum back actuated by the small motordriven exhaust pump at the right. The over-all height of the instrument is approximately $4 \frac{1}{2}$ feet. Figure 5 shows the instrument open at the top to expose the glass pressure plate that holds the negative film flat and with the vacuum plate swung out to permit loading the instrument with a sheet of sensitive paper.

Figure 6 shows the optical system of the Pleon rectifier. The lamp, shown at $A$, was apparently a mercury are of the pressure type, familiar in this country, with the mercury vapor contained in a capsule of fused-quartz. The lamp of the rectifier received from Germany was broken, and a duplicate was not obtainable. The rectifying system is not corrected chromatically, a filter, apparently of cobalt glass, forming a part of the system. The group of parts marked $B$ comprises this filter and a 


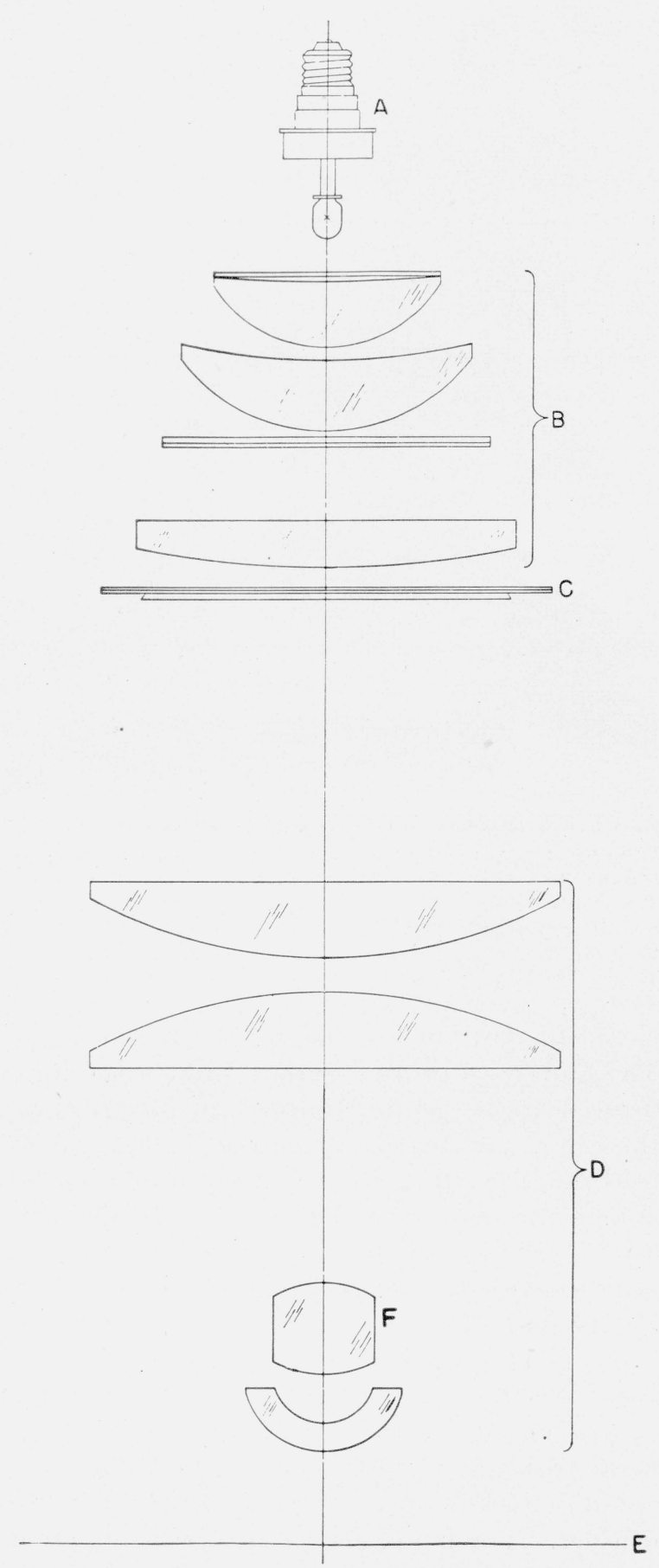

Figure 6. Optical system of the Pleon rectifier.

condensing system. At $C$ is the stage on which the negative to be copied is placed and also a variable density filter for compensating the otherwise uneven illumination of the image. This filter also failed to arrive from Germany, and an approximate filter was produced by placing a photographic plate at $C$ and exposing it by light transmitted backward through the optical system from $E$. The rectified image is produced in the plane at $E$ by the projection system marked $D$. This part of the optical system contains one detail not clearly recognizable from figure 2 . Lens $F$ appears to be double convex. Actually, however, the surface nearer $E$ is coated with black opaque paint except for an area on the axis not greater than $1 \mathrm{~mm}$ in diameter. This area, which is the limiting diaphragm of the system, is concave, the radius of curvature being approximately equal to that of the first surface of component $F$. The over-all length of the system, from the mercury capsule of the lamp to plane $E$, is approximately $110 \mathrm{~cm}$. Figure 4 shows the complete rectifier. With the distortion changing so rapidly from the center outward, it is evident that the axial point of the negative must be carefully alined with the axis of the rectifying system if the resulting print is to be distortion free or even radially symmetrical. To facilitate this adjustment an auxiliary optical system, not shown in figure 6, is provided. Immediately below stage $C$ there are four prisms by which the images, on the negative, of the four camera collimation markers can be simultaneously viewed and brought into coincidence with indexes in the rectifier. In figure $4, G$ is the eyepiece through which the markers are viewed and $H$ is a knob for throwing the prisms out of the field after the adjustment has been completed. The vacuum plate that holds the paper flat is shown at $E$. Openings, covered by doors, shown on figure 5, are also provided for inserting a paddle over the printing paper for dodging to compensate for nonuniform illumination of the terrain at the time that the exposure was made.

\section{Measurements on Pleon Lens and the Rectifier}

The conjugate distances for the rectifying lens were so adjusted that there was a reduction on the axis of $1: 0.82$. Consequently, the scale of the rectified print corresponds to an equivalent focal length of $59.1 \mathrm{~mm}$. The resulting circular print, for an angle of 65 degrees, if accurately freed from distortion, would have a radius of $126.8 \mathrm{~mm}$. For a point at the edge of the field ( $\beta=65$ degrees), the magnification incidental to the rectification is 4.58 diameters. If the magnification on the axis 
were $1: 1$, the magnification at the edge would be 5.59 diameters. The resolving power of the camera lens was measured at 5-degree intervals. The over-all resolving power of the camera lens and the rectifying system was obtained by computation, the assumption being that the rectifying lens recorded faithfully all detail in the original negative. The final resolving power for any angle (measured in the object space) therefore, is equal to the resolving power of the Pleon lens divided by the magnifying power of the rectifier for the same object ray. On the basis of such computation, the resolving power on the final print is 100 lines per millimeter on the axis, and for 65 degrees it is 4 and 6 lines, respectively, for tangential and radial lines.

In figure 7 the linear distortions are plotted for the camera lens, the rectifying unit, and the combination of the two systems. The values of the distortion for the camera lens have been multiplied by 0.82 , corresponding to the axial reduction introduced by the rectifying system before being plotted on figure 7. This having been done, the distortion for the complete process corresponding to any angular distance from the axis is found by adding the ordinates corresponding to the lens and rectifier. It will be noticed that there is a net negative distortion of $-4.34 \mathrm{~mm}$ at the edge of the resulting print after rectification. This is

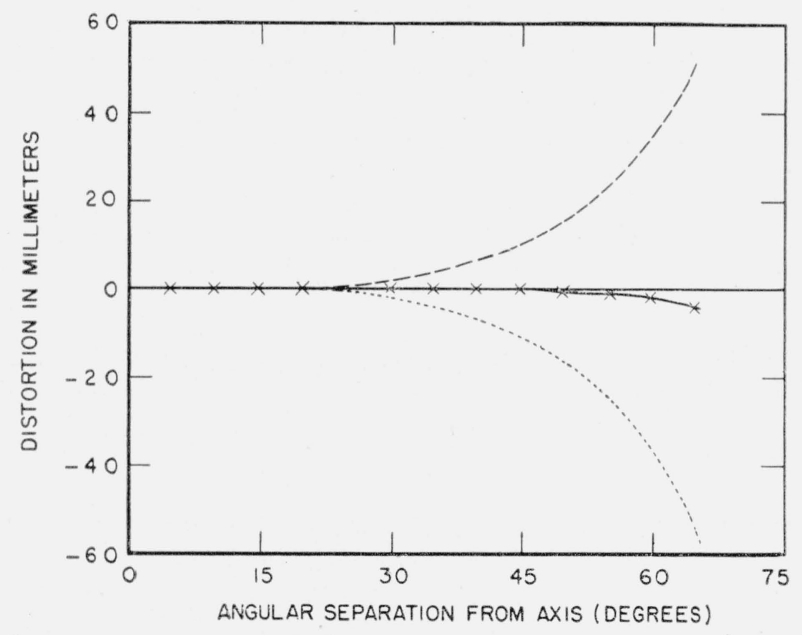

FIGURE 7. Linear distoration of camera lens, rectifying system, and final rectified print.

The dotted curve shows the linear distortion of the camera lens, multiplied by 0.82 to reduce the vaiues to the scale of the rectified print. The dashed curve shows the linear distortion of the rectifying system. The full-line curve, obtained by adding the corresponding ordinates of the first two curves, shows the residual distortion in the final print. much too great for photogrammetric purposes. However, this result should be accepted with considerable caution because there is a possibility that camera lens and rectifier were designed to be selectively paired for use, and the two instruments received for test have not had the advantage of such pairing. Nevertheless, certain general considerations stand out. The camera lens has a negative distortion of $-68.8 \mathrm{~mm}$ at the edge of the field. When the distortion is so large, it is to be expected that not only third order but many higher orders of distortion are present. If the rectifier is to accomplish its purpose in a satisfactory manner it must introduce the same orders of distortion in such magnitudes that each order of distortion in the negative is very closely compensated by a term of the same order in the rectifier. With camera lens and rectifier system so different in design, it is hardly to be expected that complete compensation for all orders can be secured.

The camera lens tested was No. 2801326, nominal focal length $72.5 \mathrm{~mm}$, and maximum aperture ratio $1: 8$. It bore the Zeiss imprint and was definitely a Zeiss product. The rectifier was No. 200701 and bore the manufacturer's code designation "rIn." Ordinarily, Zeiss products, during the war, were marked with the code "blc," but one of the German scientists now in this country affirms that the rectifier was manufactured by Zeiss, despite the code "rln."

\section{Focal Lengths}

The equivalent focal length and back focal length were found to be 72.13 and. $74.78 \mathrm{~mm}$, respectively. This value of the equivalent focal length was obtained from the approximate relation $f=d$ cot 5 degrees, where $f$ is the focal length, $d$ is the distance separating fiducial marks in the images on the negative of targets that are located at 0 and 5 degrees from the lens axis in the object space. These values of the focal length have been selected to give best average definition across the entire negative and do not necessarily correspond to those values of the focal length that give best definition on the axis. The probable errors of these determinations do not exceed $\pm 0.10 \mathrm{~mm}$.

\section{Distortion of Pleon Lens and the Rectifier}

The values of the distortion listed in table 1 are measured in millimeters and indicate the dis- 
placement of the image from its distortion-free position. A positive value indicates a displacement from the center of the plate. The probable errors for the Pleon lens data do not exceed \pm 0.02 $\mathrm{mm}$ for the range 0 to 45 degrees; beyond this range the probable error increases rapidly and may be as high as $\pm 0.20 \mathrm{~mm}$ at 65 degrees. The probable errors for the projector lens do not exceed $\pm 0.03 \mathrm{~mm}$ for the range 0 to 45 degrees; beyond this range the probable error increases rapidly and may be as high as $\pm 1.00 \mathrm{~mm}$ at 65 degrees.

TABLE 1. Distortion of the Pleon lens, rectifier, and final print from rectifier

\begin{tabular}{|c|c|c|c|c|c|}
\hline \multirow{2}{*}{$\begin{array}{l}\text { Angular } \\
\text { separation } \\
\text { from the } \\
\text { axis }\end{array}$} & \multirow{2}{*}{$\begin{array}{l}\text { Distance } \\
\text { from } \\
\text { center of } \\
\text { the Pleon } \\
\text { lens nega- } \\
\text { tive }\end{array}$} & \multirow{2}{*}{$\begin{array}{c}\text { Distortion } \\
\text { in Pleon } \\
\text { lens } \\
\text { negative }\end{array}$} & \multicolumn{3}{|c|}{$\begin{array}{l}\text { Contributions to distortion in } \\
\text { final print }\end{array}$} \\
\hline & & & $\begin{array}{c}\text { Pleon lens } \\
\text { (adjusted) }\end{array}$ & $\begin{array}{l}\text { Projection } \\
\text { lens (con- } \\
\text { tributed) }\end{array}$ & $\begin{array}{l}\text { Net dis- } \\
\text { tortion }\end{array}$ \\
\hline Degrees & $m m$ & $m m$ & $m m$ & $m m$ & $m m$ \\
\hline 0 & 0.00 & 0.00 & 0.00 & 0.00 & 0.00 \\
\hline 5 & 6.30 & .00 & .00 & +.02 & 0.02 \\
\hline 10 & 12. 64 & -.08 & -.06 & .12 & .08 \\
\hline 15 & 19. 02 & -.30 & -.24 & .29 & .05 \\
\hline 20 & 25.47 & --.78 & -.64 & .67 & .03 \\
\hline 25 & 32.00 & -1.62 & -1.32 & 1.41 & .09 \\
\hline 30 & 38.64 & -3.00 & -2.45 & 2.47 & .02 \\
\hline 35 & 45.38 & -5.12 & -4.19 & 4. 19 & .00 \\
\hline 40 & 52.20 & -8.32 & -6.80 & 6.88 & .08 \\
\hline 45 & 59.08 & -13.04 & -10.66 & 10.60 & -.06 \\
\hline 50 & 65.91 & -19.98 & -16.34 & 15.51 & -.83 \\
\hline 55 & 72.68 & $-30 \quad 16$ & -24.66 & 23.96 & -.70 \\
\hline 60 & 79.22 & -45.44 & -37.15 & 35.34 & -1.81 \\
\hline 65 & 85.39 & -68.80 & -56.26 & 51.92 & -4.34 \\
\hline
\end{tabular}

The distortion of the Pleon lens was determined in the usual manner with the aid of the precision lens-testing camera, ${ }^{6}$ with the exception that a second setting of the camera bench was necessary to measure the region from 45 to 70 degrees. For the projector lens, a halftone screen with accurately measured spacings was used as target, a deep-blue filter (tricolor C) was placed over the target, and exposure made with a moving lamp instead of the illuminator supplied with the projector. (This method was necessary because the special bulb required for the projector was not available.) The exposure was made on a Panatomic-X plate. Correspondence of the angular values reported for both lenses was accomplished by a series of interpolations, with the distance from the center of the

\footnotetext{
' I. C. Gardner and,F. A. Case, J. Research NBS 18, 449 (1937) RP984.
}

master negative made on the precision lens-testing camera serving as the connecting link. The primary purpose in effecting this correspondence was to permit the distortion of the lens and the projector to be evaluated at the same point on the negative made with the Pleon lens. Thus these values can be used in evaluating the performance of the combination, as is done in the last column of table 1.

\section{Resolving Power of the Pleon Lens and the Rectifier}

The values of the resolving power, shown in table 2, are given at 5-degree intervals from the center of the field, and, for the Pleon lens, are obtained by photographing test charts comprised of patterns of parallel lines. The series of patterns of the test charts are imaged on the negative with the lines spaced $10,15,20,29,41$, $58,81,116$, and 163 lines per millimeter. The values marked "tangential" give the number of lines per millimeter in the image on the negative of the finest pattern of the test chart that is distinctively resolved into separare lines when the lines lie perpendicular to the radius drawn from the center of the field. The row marked "radial" gives similar values for the pattern of test lines lying parallel to the radius. Because of the unusual distortion characteristics of this lens, the above series of resolving power values is valid only on the axis of the lens. Therefore, in order to avoid error, line spacings were measured in each image on the negative, and the values of resolving power computed from these measurements.

The value of the resolving power of the projection lens was not measured, but the resolving. power in a final print has been computed on the assumption that the projection lens reproduces all detail present in the original negative. Reference to table 2 shows that the distance from the axis to the image of a point at the angular distance $\beta$ from the axis is closely proportional to $\beta$. In the final image, if the distortion is eliminated, the corresponding distance is proportional to $\tan \beta$. Therefore, the instantaneous value of the magnification introduced by the projection lens, corresponding to any value $\beta$ is $1 / 1.223 \cos ^{2} \beta$, where 1.223 is the reciprocal of the axial magnification introduced by the projection lens. It is, therefore, evident that the formula 
TABLE 2. Resolving power in lines per millimeter

\begin{tabular}{|c|c|c|c|c|}
\hline \multirow{2}{*}{$\begin{array}{l}\text { Angular } \\
\text { separa- } \\
\text { tion from } \\
\text { the axis }\end{array}$} & \multicolumn{2}{|c|}{$\begin{array}{l}\text { Pleon lens } \\
\text { (measured) }\end{array}$} & \multicolumn{2}{|c|}{$\begin{array}{l}\text { Projection lens } \\
\text { (computed) }\end{array}$} \\
\hline & $\begin{array}{c}\text { Tangen- } \\
\text { tial }\end{array}$ & Radial & Tangenital & Radial \\
\hline 0 & 82 & 82 & 100 & 100 \\
\hline 5 & 82 & 82 & 100 & 100 \\
\hline 10 & 86 & 83 & 102 & 98 \\
\hline 15 & 85 & 83 & 97 & 95 \\
\hline 20 & 72 & 83 & 78 & 90 \\
\hline 25 & 59 & 81 & 59 & 81 \\
\hline 30 & 54 & 81 & 50 & 74 \\
\hline 35 & 55 & 59 & 45 & 48 \\
\hline 40 & 54 & 47 & 39 & 34 \\
\hline 45 & 57 & 34 & 35 & 21 \\
\hline 50 & 39 & 27 & 20 & 14 \\
\hline 55 & 38 & 29 & 15 & 12 \\
\hline 60 & 43 & 30 & 13 & 9 \\
\hline 65 & 18 & 29 & 4 & 6 \\
\hline 70 & -. & 31 & $\ldots$ & 4 \\
\hline
\end{tabular}

Resolving power $=1.223 r \cos ^{2} \beta$,

where $r$ is the resolving power of the Pleon lens corresponding to the value $\beta$, is the resolving power of the combination of lens and projector, assuming that no loss of detail is caused by the projection lens. It is, therefore, the maximum resolving power obtainable in the final projected print of a negative made with the Pleon lens, neglecting reductions that may result from aperture limitations. In view of aperture limitation discussed in the description of component $F$ in figure 2 , it is possible that the maximum resolving power for the rectifier may not exceed 20 lines per millimeter, which is, however, still adequate for good prints.

\section{Effect of Vignetting for the Pleon Lens}

In table 3, the vignetting of the Pleon lens is given, together with the comparable quantities for an ideal lens and for a Topogon-type lens. Let $A_{\beta}$ be the projected area of the stop opening as viewed from the object space at the angular obliquity $\beta$; and let $A_{0}$ be the corresponding area of the stop opening as viewed from the object space along the lens axis. Then the ratio $A_{\beta} / A_{0}$, on the basis of first order imagery, is equal to $\cos \beta$ for the case of the ideal lens having no vignetting. Appreciable vignetting exists in the Pleon lens as the measured value of $A_{\beta} / A_{0}$ is smaller for it than

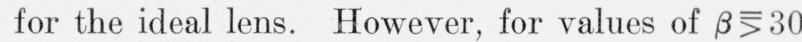
degrees, the loss of light by vignetting is much less for the Pleon lens than for the Topogon-type lens.

Table 3. Vignetting of the Pleon lens

\begin{tabular}{|c|c|c|c|}
\hline \multirow{2}{*}{$\begin{array}{l}\text { Angular } \\
\text { separa- } \\
\text { tion } \\
\text { from the } \\
\text { axis }\end{array}$} & \multicolumn{3}{|c|}{$\begin{array}{l}\text { Relative area of diaphragm stop } \\
\qquad \mathbf{A}_{\beta} / \mathbf{A}_{0}\end{array}$} \\
\hline & $\begin{array}{l}\text { Ideal lens } \\
\text { (cosine) }\end{array}$ & Pleon lens & $\begin{array}{l}\text { Topogon } \\
\text { type lens }\end{array}$ \\
\hline Degrees & & & \\
\hline 0 & 1.00 & 1.00 & 1. 00 \\
\hline 10 & 0.98 & 0.96 & 0.96 \\
\hline 20 & .94 & .87 & .87 \\
\hline 30 & .87 & .80 & .72 \\
\hline 40 & .77 & .67 & .49 \\
\hline 45 & .71 & .60 & .25 \\
\hline 50 & .64 & .52 & \\
\hline 60 & .50 & .27 & \\
\hline 65 & .42 & .05 & . \\
\hline
\end{tabular}

Figure 8 reproduces a photograph, reduced approximately 40 percent, as made with the Pleon lens by AAF personnel at Wright Field. At the peripheral parts of the lens, the full possibilities are not realized because of haze. It is obviously desirable to have ideal atmospheric conditions, and possibly to use infrared plates, if the full 130degree field is to be achieved.

Figure 9 reproduces a rectified print reduced to the same percentage as figure 8 . This probably does not represent the best possible print, because of the haze effect in the original negative and also because, as has been mentioned, the lamp and variable density filter designed to be used with the rectifier were not available.

The rectified print (fig. 9) was made by Walter R. Darling, of the National Bureau of Standards, and he is to be commended for the resourcefulness and ingenuity that he showed in obtaining so good a print with an incomplete rectifier. 


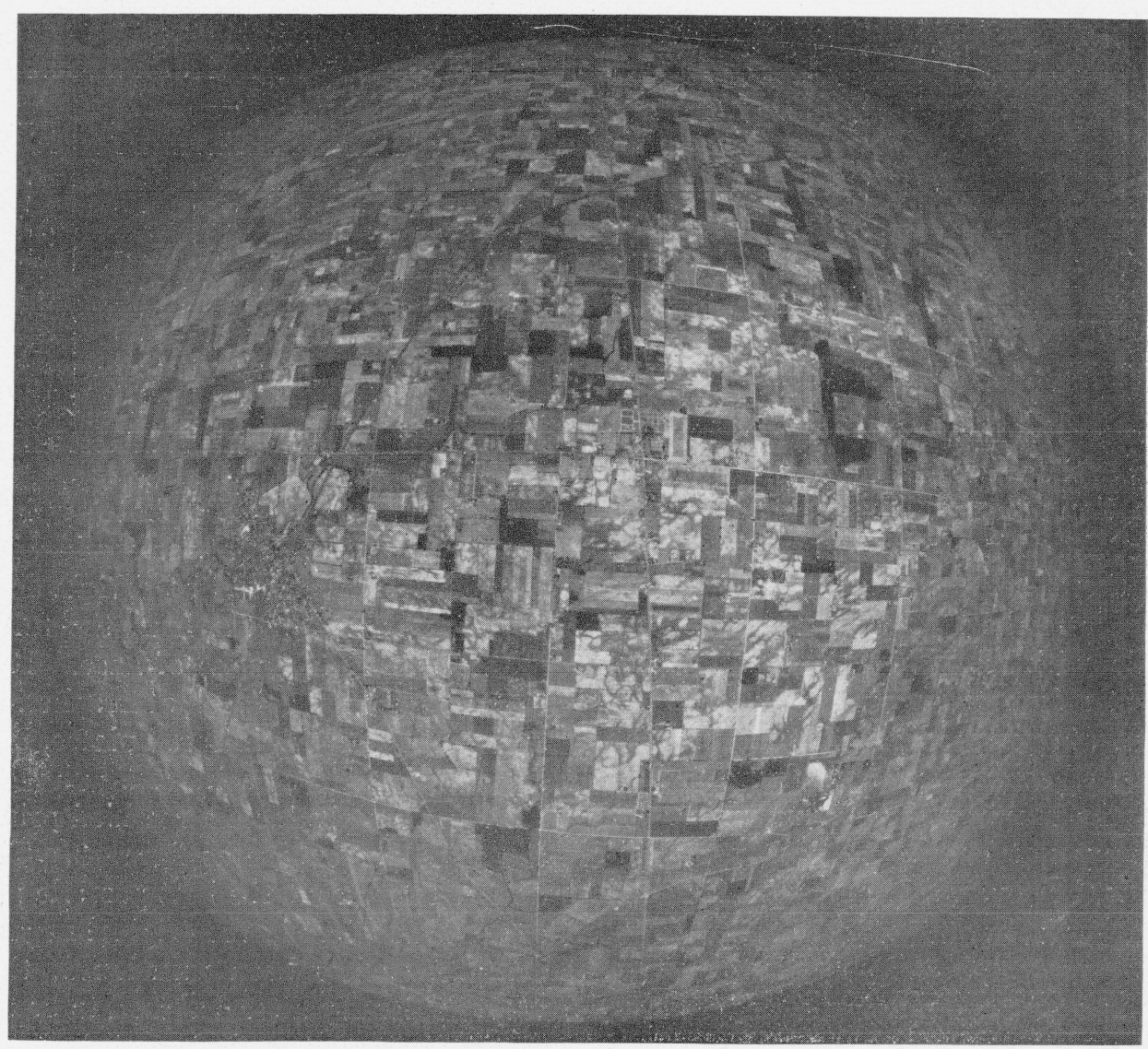

FIGURE 8. Photograph from negative exposed in airplane camera with Pleon lens. 


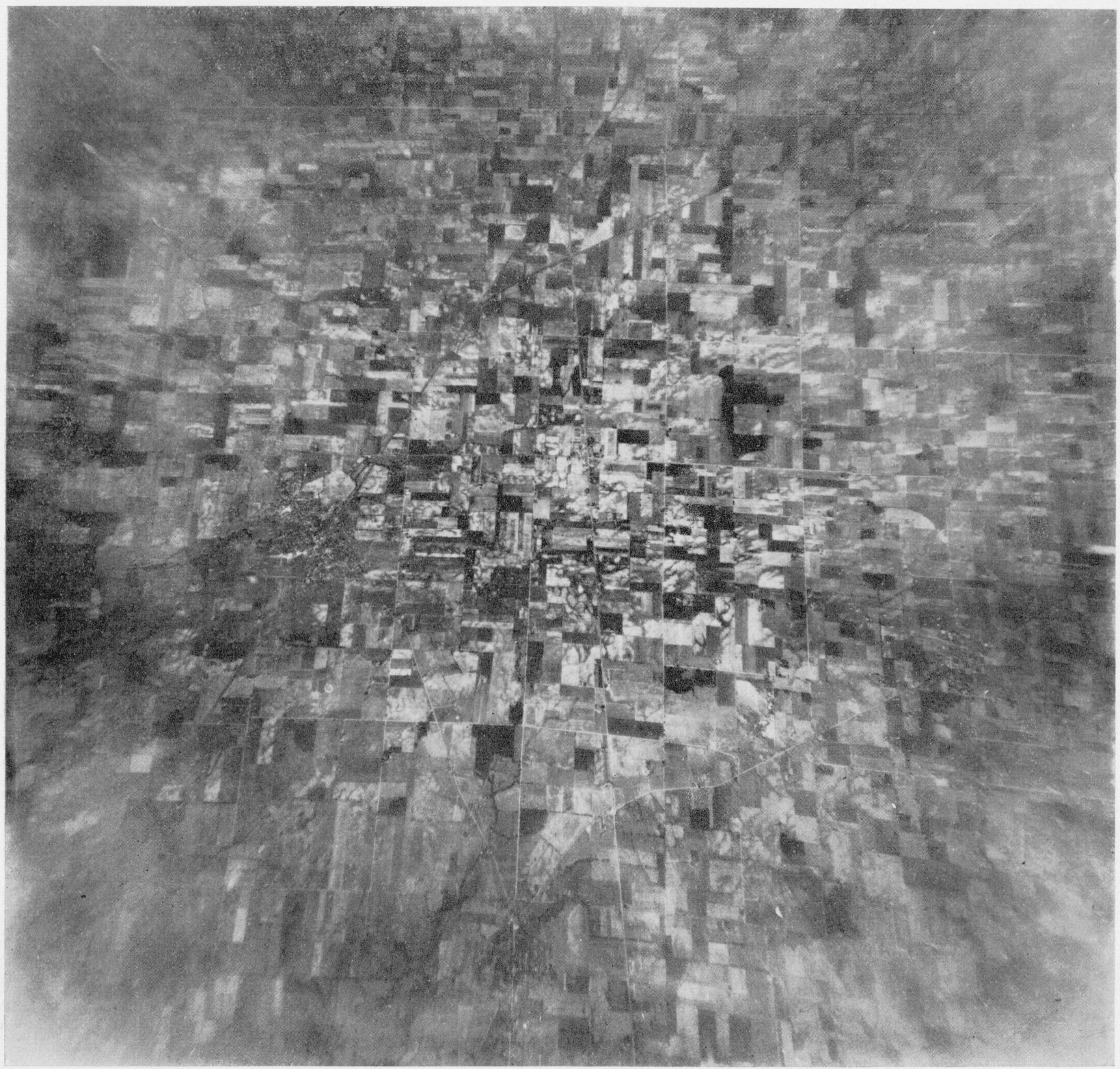

Figure 9. Rectified copy of photograph shown in figure 8.

This probably does not represent the best obtainable results, because the rectifier, when received in this country, was imperfect, the lamp being broken and the variable density filter missing. Figures 8 and 9 have been reduced in the same proportion from the original prints.

Washington, September 9, 1947. 